\title{
FLOTAC for the diagnosis of Hymenolepis spp. infection: proof-of-concept and comparing diagnostic accuracy with other methods
}

\author{
Peter Steinmann • Giuseppe Cringoli • \\ Fabrizio Bruschi • Barbara Matthys • \\ Laurent K. Lohourignon • Barbara Castagna • \\ Maria P. Maurelli • Maria E. Morgoglione • \\ Jürg Utzinger · Laura Rinaldi
}

Received: 29 December 2011 / Accepted: 13 March 2012 /Published online: 31 March 2012

(C) Springer-Verlag 2012

\begin{abstract}
Hymenolepis nana is the most common cestode parasitizing humans, yet it is under-diagnosed. We determined the optimal flotation solution (FS) for the diagnosis of this intestinal parasite with the FLOTAC method, and compared its diagnostic accuracy with an ether-concentration technique and the Kato-Katz method. Zinc sulphate (specific gravity 1.20 ) proved to be the best-performing FS. Using this
\end{abstract}

P. Steinmann $(\bowtie) \cdot J$. Utzinger

Department of Epidemiology and Public Health,

Swiss Tropical and Public Health Institute,

Socinstrasse 57, P.O. Box, 4002 Basel, Switzerland

e-mail: peter.steinmann@unibas.ch

P. Steinmann $\cdot$ B. Matthys $\cdot$ J. Utzinger

University of Basel,

Basel, Switzerland

G. Cringoli • M. P. Maurelli • M. E. Morgoglione • L. Rinaldi Department of Pathology and Animal Health, CREMOPAR

Regione Campania, University of Naples "Federico II",

Naples, Italy

F. Bruschi • B. Castagna

Department of Experimental Pathology, Faculty of Medicine,

University of Pisa,

Pisa, Italy

B. Matthys

Swiss Center for International Health,

Swiss Tropical and Public Health Institute,

Socinstrasse 57, P.O. Box, 4002 Basel, Switzerland

L. K. Lohourignon

UFR Biosciences, Université de Cocody,

Abidjan, Côte d'Ivoire
FS, we detected $65 H$. nana infections among 234 fixed fecal samples from Tajik and Sahrawi children (prevalence $27.8 \%$ ). The ether-concentration technique detected 40 infections (prevalence $17.1 \%$ ) in the same samples. Considering the combined results as a reference, the sensitivities of FLOTAC and ether-concentration were $95.6 \%$ and $58.8 \%$, respectively. The Kato-Katz method resulted in a prevalence of only $8.7 \%$. In terms of eggs per gram of stool, a significantly $(P<0.05)$ higher value was obtained with the FLOTAC and Kato-Katz techniques compared to ether-concentration. In another study carried out in China, the FLOTAC method detected six Hymenolepis diminuta infections in 302 fecal samples, whereas five samples were found positive with the Kato-Katz technique. We conclude that FLOTAC is an accurate coprodiagnostic technique for $H$. nana and $H$. diminuta, two species which join a growing list of intestinal parasites that can be reliably diagnosed by this technique.

\section{Introduction}

Hymenolepis nana, also known as dwarf tapeworm, is a small-sized cestode. Adult worms measure $15-40 \mathrm{~mm}$ and the oval parasite eggs have a size of approximately $30 \times 50 \mu \mathrm{m}$. Humans and rodents are parasitized by $H$. nana, whereas insects (beetles and fleas) act as intermediate hosts, in which infective cysticercoid larvae develop. Alternatively, the embryonated $H$. nana eggs shed in feces can directly infect humans upon ingestion. Auto-infection with eggs remaining in the intestine and hatching in situ is also possible, allowing infections to become perpetuated despite the short life span of the 
adult worm of 4-6 weeks (Schantz 1996). Adults of the related species Hymenolepis diminuta, the rat tapeworm, are much longer $(20-60 \mathrm{~cm})$, and their eggs have a round shape of approximately $60-80 \times 70-85 \mu \mathrm{m}$. The principal final hosts of H. diminuta are rodents, but human infection can occur if infected arthropods are ingested (Schantz 1996).

H. nana has a global distribution and is the most common cestode infecting humans (Schantz 1996). Highest prevalence rates are observed in warm semi-arid and arid areas among impoverished children and institutionalized persons (Schantz 1996). However, epidemiological data are scarce and only few studies have investigated the distribution of this parasite in recent years (Steinmann et al. 2010; Matthys et al. 2011). Most scientific publications are case reports among travelers, immigrants (Gualdieri et al. 2011), and adopted children (Di Lernia et al. 2004; Marseglia et al. 2007). H. diminuta is less commonly diagnosed but sporadically occurs in Asia and Europe (Wiwanitkit 2004; Patamia et al. 2010). H. nana infections often remain asymptomatic. Praziquantel and nitazoxanide are the recommended drugs (Anonymous 2007).

Diagnosis of $H$. nana and $H$. diminuta relies on the microscopic detection of eggs in fecal samples (Schantz 1996). Ethyl acetate or ether-based concentration techniques (García 2007) are recommended to increase sensitivity as egg numbers can be very low. Eggs can also be readily observed in Kato-Katz thick smears (Katz et al. 1972), a widely used diagnostic method in intestinal helminth surveys (Bergquist et al. 2009; Speich et al. 2010). Since $H$. nana eggs are small and eggs of both species are not particularly conspicuous, they might be overlooked by unsuspecting microscopists not familiar with Hymenolepis eggs. As an alternative to traditional concentration techniques, the FLOTAC apparatus has been developed (Cringoli 2006; Cringoli et al. 2010). It allows performing a series of multivalent fecal egg count (FEC) techniques for detecting and enumerating parasitic elements in the feces of animals (Rinaldi et al. 2007a, b, 2011; Duthaler et al. 2010; Cringoli et al. 2010, 2011) and humans. In human parasitology, the tool has proved to be more sensitive than the etherconcentration technique and multiple Kato-Katz thick smears for the diagnosis of soil-transmitted helminths (STHs) (Utzinger et al. 2008; Knopp et al. 2009a, b). Promising results using the FLOTAC technique have also been reported for the diagnosis of Schistosoma mansoni (Glinz et al. 2010), Dicrocoelium dendriticum (Jeandron et al. 2011), and intestinal protozoa (Becker et al. 2011; Gualdieri et al. 2011).

The objectives of the present study were to determine the optimal conditions for $H$. nana diagnosis with FLOTAC, and comparing diagnostic accuracy of FLOTAC with currently more widely used techniques for the detection of $H$. nana and $H$. diminuta eggs in fixed stool samples.

\section{Materials and methods}

Study set-up

The study was implemented in two steps. First, in a FLOTAC calibration study using a panel of nine flotation solutions (FS), we determined the FS that yielded the most accurate H. nana diagnosis (Cringoli et al. 2010). Second, the diagnostic accuracy of FLOTAC was compared with an ethyl ether-based concentration technique (García 2007; Utzinger et al. 2010) and the Kato-Katz method (Katz et al. 1972). This comparison was done using stool samples fixed in sodium acetate-acetic acid-formalin (SAF) (Marti and Escher 1990).

\section{FLOTAC calibration}

The optimal FS for $H$. nana egg detection in SAF-fixed stool samples using the FLOTAC method was determined, as described by Cringoli and co-workers (2010). In brief, two egg-positive SAF-fixed stool samples were pooled, thoroughly mixed and filtered through a wire mesh (aperture $=250 \mu \mathrm{m}$ ). The effluent suspension was divided into 54 aliquots of $2 \mathrm{ml}$ each, i.e., six replicates for each of a panel of nine FSs to be tested (see below). Each 2-ml aliquot was placed in a $15-\mathrm{ml}$ tube, centrifuged for $3 \mathrm{~min}$ at $170 \times \mathrm{g}$, and the supernatant poured off and discarded. The tube with the remaining pellet at the bottom was then filled with the respective FS until the 6-ml level mark, and slowly agitated. The suspension was transferred into one of the two $5-\mathrm{ml}$ chambers of a FLOTAC-100 apparatus using a disposable pipette. The apparatus was centrifuged at $120 \times g$ for $5 \mathrm{~min}$, and translated to separate the floating eggs from the sedimented debris. All eggs visible through the ruled window of the reading disc were enumerated under a microscope at $100 \times$ magnification.

The following nine aqueous FSs (FS1-FS9), described by Cringoli et al. (2010), were employed: FS1, sucrose and formaldehyde (specific gravity [s.g.] 1.20); FS2, sodium chloride (s.g. 1.20); FS3, zinc sulphate (s.g. 1.20); FS4, sodium nitrate (s.g. 1.20); FS5, sucrose plus mercury II iodide and potassium iodide (s.g. 1.25); FS6, magnesium sulphate (s.g. 1.28); FS7, zinc sulphate (s.g. 1.35); FS8, mercury II iodide and potassium iodide (s.g. 1.44); and FS9, zinc sulphate plus mercury II iodide and potassium iodide (s.g. 1.45). All FSs were prepared at room temperature on the day of the experiment, and their s.g. determined using a hydrometer at $20^{\circ} \mathrm{C}$.

\section{Origin of stool samples}

Overall, 234 SAF-fixed fecal samples were available to compare the diagnostic accuracy of the optimal FLOTAC 
set-up as determined in step 1 with an ethyl ether-concentration technique. Most of the stool samples $(n=215)$ were obtained from schoolchildren, aged 7-11 years, from western Tajikistan in 2009 (Matthys et al. 2011). Of note, 207 of the Tajik children had complete data records, namely FLOTAC, ether-concentration, and duplicate Kato-Katz thick smears from a single stool sample. Additionally, 19 samples were obtained in 2008 from a group of Sahrawi children, with a mean age of 9.5 years, who were hosted for summer holidays in the Tuscany region of Italy. The Sahrawi are a poor population living in the Western Sahara desert. All samples had been fixed in SAF at a ratio of approximately $1 \mathrm{~g}$ of feces and $10 \mathrm{ml}$ of fixative (Marti and Escher 1990).

H. diminuta infections were detected in SAF-fixed stool samples collected before and after anthelmintic treatment of 302 individuals of all ages and both sexes from Yunnan province, China (Steinmann et al. 2011). The focus of this diagnostic effort was the detection of STH eggs, but based on Kato-Katz thick smears prepared upon sample submission, the presence in the samples of a few infections with $H$. diminuta had been established.

\section{Diagnostic procedures}

The SAF-fixed stool samples for $H$. nana diagnosis were carefully re-suspended, filtered, and divided into two aliquots of $2 \mathrm{ml}$ each. Each aliquot was centrifuged as explained above, the supernatant poured off, and the tubes with the sediment pellets randomly assigned to diagnostic work-up using either the FLOTAC basic technique (Cringoli et al. 2010) or the ethyl ether-based concentration method (Ritchie 1948; Utzinger et al. 2010).

For the FLOTAC basic technique, tubes were filled to the $11 \mathrm{ml}$ level with the best-performing FS according to the preceding FLOTAC calibration (step 1), and slowly agitated. The suspension was then transferred into the two chambers of a FLOTAC-100 apparatus, centrifuged, and read at $100 \times$ magnification.

For the ethyl ether-based concentration technique, tubes were filled with $7 \mathrm{ml}$ of a $0.8 \%$ sodium chloride solution and $3 \mathrm{ml}$ of ethyl ether, and vigorously shaken. The tubes were then centrifuged at $240 \times g$ for $5 \mathrm{~min}$, the supernatant poured off, and the pellet re-suspended in a drop of saline solution before transfer onto a microscope slide that was examined at $100 \times$ magnification.

The samples potentially containing $H$. diminuta eggs were examined with the FLOTAC apparatus according to the same general procedures with a few modifications: FS4 was used as the focus of the study was on STHs (Utzinger et al. 2008; Knopp et al. 2009a, b, 2011) and the second aliquot was used for quality control purposes rather than the ethyl-ether concentration technique.
Ethical considerations and treatment

The study protocol in Tajikistan was approved by the Ministry of Health (reference no. 16/75-92). The protocol for the study of intestinal parasites in Sahrawi children was approved by the Ethical Committee of Azienda Ospedaliera Universitaria (AOU) Pisana, Pisa, Italy. Consent was provided by the children's parents. The study conducted in China was cleared by the ethics committee of Basel (EKBB, reference no. 294/08) and the institutional review board of the National Institute of Parasitic Diseases, Chinese Center for Disease Control and Prevention in Shanghai (reference no. 2008091701). The study is registered with Current Controlled Trials (identifier: ISRCTN47375023). Participants and the parents or legal guardians of eligible children signed a written informed consent sheet. Upon study completion, free anthelmintic treatment was offered to study participants.

\section{Statistical analysis}

For the calibration, differences between FSs were analyzed using one-way analysis of variance (ANOVA) with post hoc Fisher's least significant difference (LSD). For an assessment of the diagnostic accuracy of different techniques, egg counts were multiplied with the appropriate factor (ten for FLOTAC and ethyl etherconcentration, 24 for Kato-Katz), and the arithmetic mean number of eggs per gram of feces (EPG) calculated for each individual.

Statistical analysis was carried out using STATA version 10.0 (Stata Corp.; College Station, TX, USA). The statistical differences between the mean EPG values were analyzed using ANOVA (generalized linear model [GLM] for repeated measures) in conjunction with the Bonferroni test for post hoc comparison. In addition, a likelihood ratio test (LRT) of the equality of the coefficients of variation of $k$ normally distributed populations was performed using the software developed by Verril and Johnson (2007).

\section{Results}

FLOTAC calibration

The mean $H$. nana EPG value calculated for each of the nine FSs is shown in Fig. 1, along with the coefficient of variation (CV). H. nana eggs were detected with each FS. The best result, in terms of highest mean FEC (462.7 EPG) and lowest CV (6.5\%), was obtained using FS3, i.e., zinc 
Fig. 1 Mean number of Hymenolepis nana eggs per gram of stool (EPG) and coefficient of variation $(\mathrm{CV})$ in human stool samples when using FLOTAC with nine different flotation solutions (FSs; six replicates per FS)

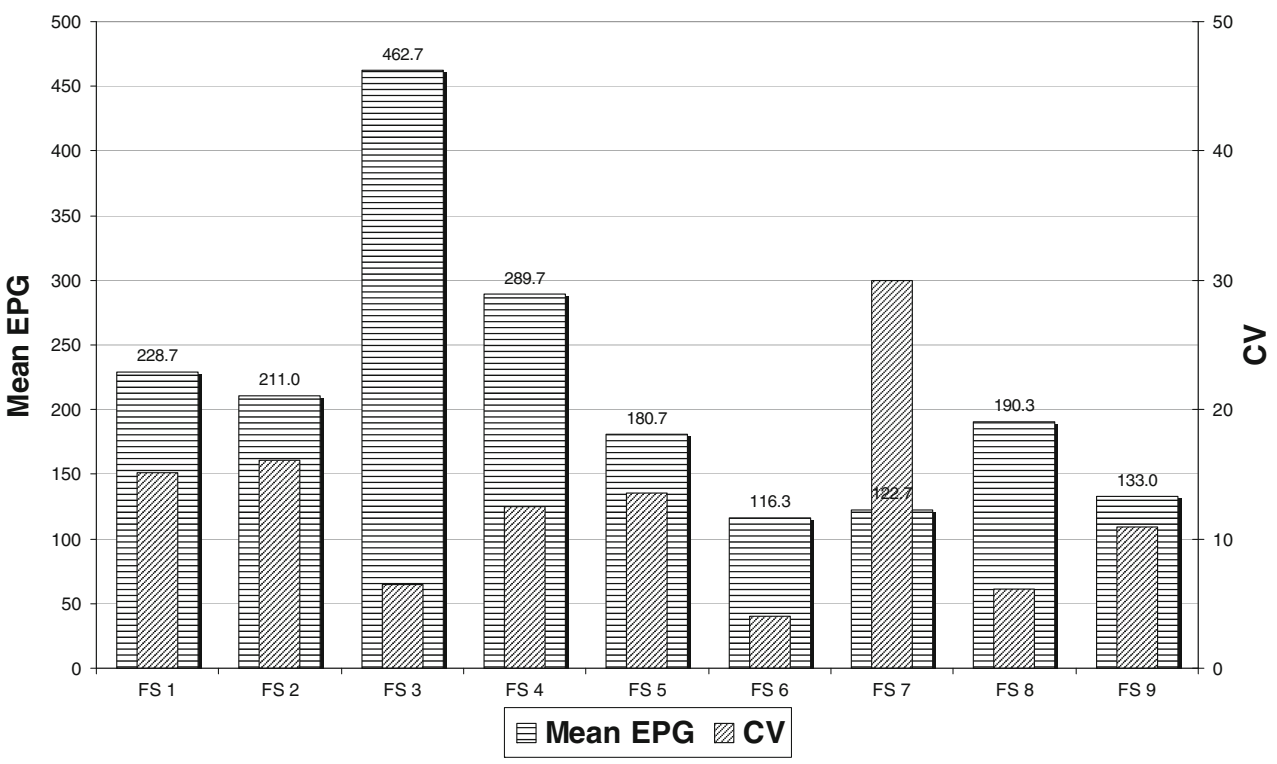

sulphate (s.g. 1.20). Hence, for the subsequent comparison of diagnostic accuracy, FS3 was used.

\section{Diagnostic accuracy}

Using FLOTAC with FS3, H. nana eggs were detected in 65 out of the 234 stool samples, translating into a prevalence of $27.8 \%$. Employing the ethyl ether-concentration technique on the same stool samples, considerably fewer $H$. nana infections were detected $(n=40,17.1 \%)$. Both methods combined revealed 68 infections $(29.1 \%)$. Considering these combined results as a reference, the sensitivity of the FLOTAC and the ethyl ether-concentration techniques for the diagnosis of $H$. nana were $95.6 \%$ and $58.8 \%$, respectively (difference $P=0.002$ ).

The results, stratified by sample origin and diagnostic technique, are shown in Table 1. Among the 215 Tajik samples, $58(27.0 \%)$ were positive for $H$. nana according to the FLOTAC technique (sensitivity: $95.1 \%$ ), while only $37(17.2 \%)$ samples were positive using the ethyl ether-based concentration technique (sensitivity: $60.7 \%$ ). Among the 207 samples screened with the Kato-Katz method, $18 \mathrm{H}$. nana infections, including four that were missed by the FLOTAC technique, were observed (prevalence: $8.7 \%$ ). For the 19 Sahrawi samples, seven $(36.8 \%)$ were found positive with the FLOTAC technique (sensitivity: $100 \%$ ), but only three $(15.8 \%)$ were positive using the ethyl ether-based concentration (sensitivity: $42.9 \%$ ).

The mean number of $H$. nana eggs detected with FLOTAC (mean among positives: 617.4 EPG) was significantly higher $(P<0.05)$ than the numbers counted after ethyl ether-concentration (mean among positives: 58.2 EPG). Diagnosis with the Kato-Katz method resulted in a mean of 660.7 EPG among the infected (Table 1).

A total of eight $H$. diminuta-positive samples from six individuals were identified with the Kato-Katz, FLOTAC, or both methods combined among the samples from China.
Table 1 Hymenolepis nana prevalence and fecal egg count (FEC, expressed as eggs per gram of stool (EPG)) in human stool samples from Tajik and Sahrawi children, stratified by diagnostic technique

n.a., not available

*Significant differences for different letters $(\mathrm{a}, \mathrm{b})(P<0.05)$

\begin{tabular}{|c|c|c|c|c|}
\hline \multirow[t]{2}{*}{ Method } & \multirow[t]{2}{*}{ No. of positive results (\%) } & \multicolumn{3}{|c|}{ FEC among positives, in EPG* } \\
\hline & & Arithmetic mean & Median & Interquartile range \\
\hline \multicolumn{5}{|l|}{ FLOTAC with FS3 } \\
\hline $\begin{array}{l}\text { - Total }(n=234) \\
\text { - Tajikistan }(n=215) \\
\text { - Sahrawi }(n=19)\end{array}$ & $\begin{array}{r}65(27.8) \\
58(27.0) \\
7(36.8)\end{array}$ & $\begin{array}{r}617.4^{\mathrm{a}} \\
\text { n.a. } \\
\text { n.a. }\end{array}$ & 17.5 & 147.8 \\
\hline \multicolumn{5}{|c|}{ Ethyl ether-concentration } \\
\hline $\begin{array}{l}\text { - Total }(n=234) \\
\text { - Tajikistan }(n=215) \\
\text { - Sahrawi }(n=19)\end{array}$ & $\begin{array}{r}40(17.1) \\
37(17.2) \\
3(15.8)\end{array}$ & $\begin{array}{c}58.2^{\mathrm{b}} \\
\text { n.a. } \\
\text { n.a. }\end{array}$ & 11.0 & 24.0 \\
\hline \multicolumn{5}{|l|}{ Kato-Katz } \\
\hline - Tajikistan $(n=207)$ & $18(8.7)$ & $660.7^{\mathrm{a}}$ & 558 & 459 \\
\hline
\end{tabular}


Three cases were tested positive with both methods; eggs were only seen on Kato-Katz thick smears prepared from two samples, the remaining three samples were only positive with the FLOTAC technique.

\section{Discussion}

The FS is a key determinant of the sensitivity of any copromicroscopic technique that is based on flotation (Cringoli 2006; Cringoli et al. 2010). In the present study, we found that zinc sulphate (s.g. 1.20; designated FS3 according to a standard protocol) is the optimal FS for detecting $H$. nana eggs with FLOTAC out of a panel of nine tested FSs. Indeed, FS3 resulted in the highest mean FEC (462.7 EPG) and the smallest CV (6.5\%). We also found that FLOTAC shows a higher sensitivity than the widely used ethyl ether-based concentration technique (sensitivity: $95.6 \%$ versus $58.8 \%$ ). Perhaps surprisingly, the Kato-Katz method detected only about half as many infections than the ethyl ether concentration technique. FLOTAC not only showed a higher sensitivity than the ether-concentration method, it also resulted in a several-fold higher number of eggs counted in a given amount of stool (FLOTAC, FEC= 617.4 EPG; ether-concentration technique, $\mathrm{FEC}=58.2$ EPG). Somewhat higher FECs have been observed when comparing the FLOTAC method with an ether-concentration method for the diagnosis of other nematodes and trematodes (Utzinger et al. 2008; Glinz et al. 2010). Interestingly, $H$. nana FECs derived from the FLOTAC and the Kato-Katz techniques were comparable, which is in contrast to previous investigations pertaining to STHs, which revealed considerably higher FECs for Kato-Katz rather than FLOTAC (Utzinger et al. 2008; Knopp et al. 2009a, b).

In the light of the available evidence, FS2 and FS4 are the recommended FSs for surveys pertaining to intestinal helminths. Indeed, FS4 has proved to perform best for STH diagnosis in several studies and was the second bestperforming FS for $H$. nana diagnosis according to the current calibration study. Moreover, FS4 also shows promise for the diagnosis of $H$. diminuta. The latter parasite is only rarely diagnosed in humans and the detection of six cases among a sample of 302 individuals in Yunnan province, China (prevalence of approximately $2 \%$ ), is noteworthy. To date, only around 500 cases of human infections with this parasite have been reported in the scientific literature, albeit this tapeworm is suspected to be considerably more common in humans (Schantz 1996; Wiwanitkit 2004).

Based on the growing evidence of its diagnostic competitiveness with more traditional methods, FLOTAC is gaining in importance as a new or complementary tool in parasitological surveys among humans. Examples include epidemiological surveys and anthelmintic drug efficacy studies in Zanzibar (Knopp et al. 2009b, 2011), and comparative diagnostic studies in Côte d'Ivoire (Glinz et al. 2010), Ethiopia (Habtamu et al. 2011), and Kyrgyzstan (Jeandron et al. 2011). Moreover, in a recent investigation of intestinal parasites among immigrants in Naples, Italy, it was demonstrated that the FLOTAC was more sensitive than an ethyl acetate-based concentration technique for helminths and intestinal protozoa (Gualdieri et al. 2011). The FLOTAC technique has also been employed for point-of-care diagnosis and results obtained thus far warrant follow-up studies (Coppola et al. 2010).

We conclude that FLOTAC is an efficient coprodiagnostic technique for detecting intestinal parasite infections. Specifically, $H$. nana and $H$. diminuta can be added to the growing list of human intestinal parasites which are reliably diagnosed with the FLOTAC technique. These results should be confirmed by other groups in areas where hymenolepiasis is endemic.

Acknowledgements The efforts of the staff at CREMOPAR who provided technical support to this work are acknowledged.

\section{References}

Anonymous (2007) Drugs for parasitic infections. Med Lett 5(Suppl): e1-e15

Becker SL, Lohourignon LK et al (2011) Comparison of the Flotac400 dual technique and the formalin-ether concentration technique for diagnosis of human intestinal protozoon infection. J Clin Microbiol 49:2183-2190

Bergquist R, Johansen MV, Utzinger J (2009) Diagnostic dilemmas in helminthology: what tools to use and when? Trends Parasitol 25:151-156

Coppola G, Gualdieri L et al (2010) Naples immigrants and parasites. Parassitologia 52:33-36

Cringoli G (2006) FLOTAC, a novel apparatus for a multivalent faecal egg count technique. Parassitologia 48:381-384

Cringoli G, Rinaldi L et al (2010) FLOTAC: new multivalent techniques for qualitative and quantitative copromicroscopic diagnosis of parasites in animals and humans. Nat Protoc 5:503-515

Cringoli G, Rinaldi L et al (2011) Ancylostoma caninum: calibration and comparison of diagnostic accuracy of flotation in tube, McMaster and FLOTAC in faecal samples of dogs. Exp Parasitol 128:32-37

Di Lernia V, Ricci C, Albertini G (2004) Skin eruption associated with Hymenolepis nana infection. Int J Dermatol 43:357-359

Duthaler U, Rinaldi L et al (2010) Fasciola hepatica: comparison of the sedimentation and FLOTAC techniques for the detection and quantification of faecal egg counts in rats. Exp Parasitol 126:161166

García LS (2007) Diagnostic medical parasitology. ASM Press, Washington

Glinz D, Silué KD et al (2010) Comparing diagnostic accuracy of Kato-Katz, Koga agar plate, ether-concentration, and FLOTAC for Schistosoma mansoni and soil-transmitted helminths. PLoS Negl Trop Dis 4:e754

Gualdieri L, Rinaldi L et al (2011) Intestinal parasites in immigrants in the city of Naples (southern Italy). Acta Trop 117:196-201 
Habtamu K, Degarege A et al (2011) Comparison of the Kato-Katz and FLOTAC techniques for the diagnosis of soil-transmitted helminth infections. Parasitol Int 60:398-402

Jeandron A, Rinaldi L et al (2011) Human infections with Dicrocoelium dendriticum in Kyrgyzstan: the tip of the iceberg? J Parasitol 97:1170-1172

Katz N, Chaves A, Pellegrino J (1972) A simple device for quantitative stool thick-smear technique in schistosomiasis mansoni. Rev Inst Med Trop São Paulo 14:397-400

Knopp S, Glinz D et al (2009a) FLOTAC: a promising technique for detecting helminth eggs in human faeces. Trans R Soc Trop Med Hyg 103:1190-1194

Knopp S, Rinaldi L et al (2009b) A single FLOTAC is more sensitive than triplicate Kato-Katz for the diagnosis of low-intensity soiltransmitted helminth infections. Trans R Soc Trop Med Hyg 103:347-354

Knopp S, Speich B et al (2011) Diagnostic accuracy of Kato-Katz and FLOTAC for assessing anthelmintic drug efficacy. PLoS Negl Trop Dis 5:e1036

Marseglia GL, Marseglia A et al (2007) Chronic urticaria caused by Hymenolepis nana in an adopted girl. Allergy 62:821-822

Marti H, Escher E (1990) SAF - an alternative fixation solution for parasitological stool specimens. Schweiz Med Wochenschr 120:1473-1476 (in German)

Matthys B, Bobieva M et al (2011) Prevalence and risk factors of helminths and intestinal protozoa infections among children from primary schools in western Tajikistan. Parasit Vectors 4:195

Patamia I, Cappello E et al (2010) A human case of Hymenolepis diminuta in a child from eastern Sicily. Korean J Parasitol 48:167-169

Rinaldi L, Calabria G et al (2007a) Crenosoma vulpis in dog: first case report in Italy and use of the FLOTAC technique for copromicroscopic diagnosis. Parasitol Res 101:1681-1684
Rinaldi L, Russo T et al (2007b) Passalurus ambiguus: new insights into copromicroscopic diagnosis and circadian rhythm of egg excretion. Parasitol Res 101:557-561

Rinaldi L, Coles GC et al (2011) Calibration and diagnostic accuracy of simple flotation, McMaster and FLOTAC for parasite egg counts in sheep. Vet Parasitol 177:345-352

Ritchie LS (1948) An ether sedimentation technique for routine stool examinations. Bull US Army Med Dep 8:326

Schantz PM (1996) Tapeworms (cestodiasis). Gastroenterol Clin N Am 25:637-653

Speich B, Knopp S et al (2010) Comparative cost assessment of the Kato-Katz and FLOTAC techniques for soil-transmitted helminth diagnosis in epidemiological surveys. Parasit Vectors 3:71

Steinmann P, Usubalieva J et al (2010) Rapid appraisal of human intestinal helminth infections among schoolchildren in Osh oblast, Kyrgyzstan. Acta Trop 116:178-184

Steinmann P, Utzinger J et al (2011) Efficacy of single-dose and tripledose albendazole and mebendazole against soil-transmitted helminths and Taenia spp.: a randomized controlled trial. PLoS One 6:e25003

Utzinger J, Rinaldi L et al (2008) FLOTAC: a new sensitive technique for the diagnosis of hookworm infections in humans. Trans R Soc Trop Med Hyg 102:84-90

Utzinger J, Botero-Kleiven S et al (2010) Microscopic diagnosis of sodium acetate-acetic acid-formalin-fixed stool samples for helminths and intestinal protozoa: a comparison among European reference laboratories. Clin Microbiol Infect 16:267-273

Verril, Johnson (2007) Statistical services at the forest products laboratory. Madison, WI, USA. Available at: http://www.fpl.fs.fed.us/ documnts/fplrp/fpl_rp638.pdf

Wiwanitkit V (2004) Overview of Hymenolepis diminuta infection among Thai patients. MedGenMed 6:7 